\title{
Zener transitions between dissipative Bloch bands
}

\author{
Xian-Geng Zhao and W.-X. Yan* \\ CCAST (World Laboratory) P.O. Box 8730, Beijing 100080, China \\ Institute of Applied Physics and Computational Mathematics, \\ P.O. Box 8009, Beijing 100088, China \\ * Institute of Theoretical Physics, Academia Sinica, P.O. Box 2735, Beijing 100080, China \\ Daniel W. Hone \\ Institute for Theoretical Physics, University of California, Santa Barbara, CA 93106
}

(April 18, 2018)

\begin{abstract}
Within a two-band tight binding model, we investigate the dynamics of electrons with Markoffian dephasing under the influence of static electric fields. With the help of both numerical and analytic calculations we find that the dephasing ultimately takes electrons which are initially located in one miniband to equal population of the two minibands, instead of undergoing persistent Rabi flop, as they do in the absence of scattering. Miniband localization is wholly destroyed by the intervention of dephasing. We also obtain the effective decay time for the approach to equal band populations under conditions of small interband communication and in the long-time limit, through a perturbative calculation. The decay rate shows characteristic sharp peaks at values of the parameters which give Zener resonances.
\end{abstract}

PACS numbers: 71.70.Ej, 73.40.Gk, 73.20.Dx

\section{INTRODUCTION}

Recently there has been intensive investigation ${ }^{1-7}$ of the influence of external electric fields on semiconductor superlattices. The availability of high-quality semiconductor superlattices, the possible practical application of new phenomena, and the surge of new interesting theoretical predictions have attracted both theoretical and experimental attention to this active field. An important example is Bloch oscillatione (BO), one of the earliest predictions of the band theory of solids. Its observation in standard crystalline solids has been prevented 3 by excessive scattering under all practical conditions. But in semiconductor superlattices BOs have not only been observed experimentally $\|_{\text {hut have }}$ been further exploited to make an extremely fast emitter of electromagnetic radiation - the "Bloch oscillator" 3. Many other new phemomena have been predicted theoretically and/or observed experimentally, including negative differential

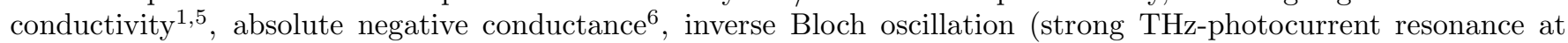
the Bloch frequency) 1 , dynamic localization 13 , band collapse 14 16, band suppression 17, fractional Wannier-Stark Ladders 18 , and multi-photon absorption 19 ,

From a theoretical point of view the simplest models involve only a single miniband. But these miss all interesting interband phenomena, which are captured most simply in a two band tight binding model first introduceded by Fukuyama, Bari and Fogedby. This medel contains the essential physics of interband transitions, and can be compared in practice with realistic situations 2123 where only a single pair of bands is important. Rotvig, Jauho and Smith 24,25 have studied coherent transport of one-dimensional semiconductor superlattices within this model. They found that coherent oscillation between the minibands can occur at special values of the applied field, where there are avoided crossings of the two interpenetrating Wannier-Stark Ladders (WSL) arising essentially from different band\$24. These are the so-called Zener resonances. For electric field values between Zener resonances there are stable plateaus, where the relative population of the two bands is only weakly dependent on time. In each plateau there are small amplitude Bloch oscillations. The number of these, $N$, corresponds to the $N$ th Zener resonance 24 (labelled with decreasing field, so that the $N$ th resonance arises from aligning adjacent WSL levels in quantum wells $N$ lattice spacings apart).

In this paper, using the same model, we study the effects of scattering from lattice imperfections on the dynamics of electrons, using a stochastic Liouville equation for the density matrix, with Markoffian dephasing. By means of both analytic and numerical calculations we analyse the effects of scattering and miniband structure parameters-op the time evolution of $\rho_{-}$, the difference in electron population of the two minibands. In contrast to previous work 24.25 , in which the single quasimomentum component $\rho_{-}(k, t)$ was studied, we focus on the trace quantity $\rho_{-}(t)=\sum_{k} \rho_{-}(k, t)$. There are two reasons for this choice. The first is that the temporal evolution of the sum over all quasimomenta is of direct physical interest, describing the dynamics of the full set of electrons. The individual $\rho_{-}(k, t)$ depend not only on time but on quasimomentum $k$, so we should integrate $\rho_{-}(k, t)$ over the whole Brillouin zone. The other is that, with scattering, $k$ is no longer a good quantum number - even in a suitable transverse gauge (see Ref. 22 and Eqs. 
(17) - (19) below), where $k$ is a good quantum number in the perfect lattice. We find that with no dissipation $\rho_{-}(t)$ periodically oscillates between 1 and $-\rho_{m}$ ("Rabi flop" between the initial condition of all electrons in one band and a fixed maximum exchange of a fraction of that population with the other band: $0<\rho_{m}<1$ ) at avoided crossings, whereas when dephasing is introduced, the electrons initially assumed to be located in one miniband will ultimately be distributed equally between the two bands (band localization will be completely destroyed).

In section II, we present the model and derive the equation of motion in $k$ space. Section III provides numerical analysis of the effects of the single relaxation rate and of the miniband parameters on the Zener resonances. Section IV contains the perturbative calculation of the density matrix and its limiting long-time behavior. We make some concluding remarks in section $\mathrm{V}$.

\section{MODEL}

We consider the standard tight-binding model of a two-band system in a static electric field E. The model Hamiltonian 20 can be written as

$$
\begin{aligned}
H= & \sum_{n}\left[\left(\Delta_{a}+n \omega_{B}\right) a_{n}^{\dagger} a_{n}+\left(\Delta_{b}+n \omega_{B}\right) b_{n}^{\dagger} b_{n}\right. \\
& -\left(W_{a} / 4\right)\left(a_{n+1}^{\dagger} a_{n}+h . c .\right)+\left(W_{b} / 4\right)\left(b_{n+1}^{\dagger} b_{n}+\text { h.c. }\right) \\
& \left.+e E R\left(a_{n}^{\dagger} b_{n}+b_{n}^{\dagger} a_{n}\right)\right] .
\end{aligned}
$$

Here the subscripts label the lattice sites and the lower and upper minibands are designated by symbols $a$ and $b$, respectively. We have introduced the notation $\omega_{B} \equiv e E d$ for the Bloch frequency, which will appear often below. The first two terms describe the site energies of the Wannier states in the presence of the electric field, and $W_{a, b}$ are the widths of the isolated $(E=0)$ minibands induced by nearest neighbor hopping: $\epsilon^{a, b}(k)=\Delta_{a, b} \mp\left(W_{a, b} / 2\right) \cos (k d)$, where $d$ is the lattice constant. The last term is the on-site electric dipole coupling between minibands; $e R$ is the corresponding dipole moment. This Hamiltonian does neglect Coulomb interactions and electric dipole elements between Wannier states on different sites, but it contains the essential physics for the problem2.222425. Note that the hopping parameters $W_{a, b}$ are written here with opposite signs, so that with both parameters positive the band structure at $E=0$ is of the standard nearly free electron character, with direct band gaps at the zone boundary. But the calculation to follow is valid for arbitrary signs of the parameters.

It is easily showned.22 that the exact spectrum of $H$ is two interpenetrating Wannier-Stark Ladders. But what do the corresponding states represent in terms of the occupation of the original bands as a function of time, and what is the influence of scattering? For vanishing dipole matrix element between bands, $R=0$, there is no interband mixing. Each of the two bands gives rise to a single WSL. Clearly, when the electric field amplitude is such that the ladders become degenerate, even small values of $R$ lead to strong interband mixing. The crossing of the ladders is "avoided" by any finite $R$, and the behavior at those avoided crossings (the Zener resonances) is of particular importance and interest.

We start by defining the density matrix in the representation of the two bands,

$$
\rho(t)=\sum_{i j m n} \rho_{m n}^{i j} \xi_{m}^{i \dagger} \xi_{n}^{j}
$$

where $i, j=1$ or 2 are band indices: $\xi_{m}^{1 \dagger}\left(\xi_{m}^{1}\right)$ and $\xi_{m}^{2 \dagger}\left(\xi_{m}^{2}\right)$ designate $a_{m}^{\dagger}\left(a_{m}\right)$ and $b_{m}^{\dagger}\left(b_{m}\right)$, respectively. Within a constant relaxation rate approximation 26 , the density matrix $\rho(t)$ satisfies the following stochastic Liouville equation (SLE) (we set $\hbar=1$ throughout this paper),

$$
i \frac{d \rho}{d t}=[H, \rho(t)]-i \Gamma \rho(t) .
$$

Here $\Gamma \rho$ describes the relaxation of the off-diagonal elements of $\rho$ through dephasing:

$$
\Gamma \rho=\sum_{i j m n}\left(1-\delta_{i j} \delta_{m n}\right)\left(\alpha_{i j} \xi_{m}^{i \dagger} \xi_{n}^{j}\right)
$$

The utility of this simplest form of the SLE has been discussed by Kenkre and collaborators (see Ref. 26 and references therein). The parameters $\alpha_{i j}$ measure the loss of phase coherence between sites, or the scattering lifetime of band states 
labeled by quasimomentum. The relaxation to zero of the lattice site off-diagonal elements_of $\rho$ is then effectively an infinite temperature approximation. The finite temperature corrections are essential26.27 to understanding the current response to an electric field, a response which vanishes in the absence of a difference in thermal equilibrium population between states of different energy, but those corrections sheyld not play a major role in the phenomena studied here. We note that in this limit there is no need to distinguish27 between the relaxation rates of energy and momentum.

Since we are interested in the dynamics of occupation of various band states, it is convenient to work in a wave vector basis, by Fourier transforming the density matrix. In general, since $\rho_{m n}$ is not translationally invariant (a function only of $m-n)$, we have a full set $\rho_{k q}^{i j}=\sum_{m n} \rho_{m n}^{i j}(t) \exp [-i k m+i q n]$ of Fourier components. But we will be interested in the wave vector diagonal band occupation numbers $\rho_{k k}^{i j}(t) \equiv \rho^{i j}(k, t)$. These evolve according to the corresponding Fourier transform of the SLE:

$$
\begin{aligned}
i \frac{\partial}{\partial t} \rho^{11}(k, t)= & i \omega_{B} \frac{\partial}{\partial k} \rho^{11}(k, t)-e E R\left[\rho^{12}(k, t)-\rho^{21}(k, t)\right] \\
& -i \alpha_{11} \rho^{11}(k, t)+i \alpha_{11} \int_{0}^{2 \pi} \frac{d k^{\prime}}{2 \pi} \rho^{11}\left(k^{\prime}, t\right) \\
i \frac{\partial}{\partial t} \rho^{22}(k, t)= & i \omega_{B} \frac{\partial}{\partial k} \rho^{22}(k, t)+e E R\left[\rho^{12}(k, t)-\rho^{21}(k, t)\right] \\
& -i \alpha_{22} \rho^{22}(k, t)+i \alpha_{22} \int_{0}^{2 \pi} \frac{d k^{\prime}}{2 \pi} \rho^{22}\left(k^{\prime}, t\right) \\
i \frac{\partial}{\partial t} \rho^{12}(k, t)= & i \omega_{B} \frac{\partial}{\partial k} \rho^{12}(k, t)-e E R\left[\rho^{11}(k, t)-\rho^{22}(k, t)\right] \\
& +(\Delta-W \cos k) \rho^{12}(k, t)-i \alpha_{12} \rho^{12}(k, t) \\
i \frac{\partial}{\partial t} \rho^{21}(k, t)= & i \omega_{B} \frac{\partial}{\partial k} \rho^{21}(k, t)+e E R\left[\rho^{11}(k, t)-\rho^{22}(k, t)\right] \\
& -(\Delta-W \cos k) \rho^{21}(k, t)-i \alpha_{21} \rho^{21}(k, t)
\end{aligned}
$$

where we have adopted the notation $\Delta \equiv \Delta_{a}-\Delta_{b}$ and $W \equiv\left(W_{a}+W_{b}\right) / 2$. By introducing

$$
\begin{array}{r}
\rho_{+}(k, t)=\rho^{11}(k, t)+\rho^{22}(k, t), \\
\rho_{-}(k, t)=\rho^{11}(k, t)-\rho^{22}(k, t), \\
\rho_{+-}(k, t)=\rho^{12}(k, t)+\rho^{21}(k, t), \\
\rho_{-+}(k, t)=i\left[\rho^{21}(k, t)-\rho^{12}(k, t)\right],
\end{array}
$$

and, for simplicity, taking $\alpha_{11}=\alpha_{22}=\alpha_{1}, \quad \alpha_{12}=\alpha_{21}=\alpha_{2}$ to reduce the number of parameters in the theory, we obtain

$$
\begin{gathered}
\frac{\partial}{\partial t} \rho_{+}(k, t)-\omega_{B} \frac{\partial}{\partial k} \rho_{+}(k, t)=-\alpha_{1}\left[\rho_{+}(k, t)-\int_{0}^{2 \pi} \frac{d k^{\prime}}{2 \pi} \rho_{+}\left(k^{\prime}, t\right)\right] \\
\frac{\partial}{\partial t} \rho_{-}(k, t)-\omega_{B} \frac{\partial}{\partial k} \rho_{-}(k, t)=-2 e E R \rho_{-+}(k, t)-\alpha_{1}\left[\rho_{-}(k, t)-\int_{0}^{2 \pi} \frac{d k^{\prime}}{2 \pi} \rho_{-}\left(k^{\prime}, t\right)\right] \\
\frac{\partial}{\partial t} \rho_{+-}(k, t)-\omega_{B} \frac{\partial}{\partial k} \rho_{+-}(k, t)=(\Delta-W \cos k) \rho_{-+}(k, t)-\alpha_{2} \rho_{+-}(k, t) \\
\frac{\partial}{\partial t} \rho_{-+}(k, t)-\omega_{B} \frac{\partial}{\partial k} \rho_{-+}(k, t)=-(\Delta-W \cos k) \rho_{+-}(k, t)+2 e E R \rho_{-}(k, t)-\alpha_{2} \rho_{-+}(k, t)
\end{gathered}
$$


The equation for $\rho_{+}(k, t)$ describes particle conservation. It is decoupled from the others, and we will ignore it in the following discussion. The equations for $\rho_{-}(k, t), \rho_{+-}(k, t)$, and $\rho_{-+}(k, t)$ can be reduced to the following ordinary differential equations in an accelerated basis $28, k(t)=k-\omega_{B} t$ or, equivalently, in the transverse or vector gauge discussed above and in Ref. 22,

$$
\begin{gathered}
\frac{d}{d t} X(k, t)=-2 e E R Z(k, t)-\alpha_{1}\left[X(k, t)-X_{0}(t)\right], \\
\frac{d}{d t} Y(k, t)=\left[\Delta-W \cos \left(k-\omega_{B} t\right)\right] Z(k, t)-\alpha_{2} Y(k, t), \\
\frac{d}{d t} Z(k, t)=-\left[\Delta-W \cos \left(k-\omega_{B} t\right)\right] Y(k, t)+2 e E R X(k, t)-\alpha_{2} Z(k, t) .
\end{gathered}
$$

Here $X(k, t)=\rho_{-}\left(k-\omega_{B} t, t\right), Y(k, t)=\rho_{+-}\left(k-\omega_{B} t, t\right), Z(k, t)=\rho_{-+}\left(k-\omega_{B} t, t\right)$, and $X_{0}(t)=\int_{0}^{2 \pi} X(k, t) d k / 2 \pi=$ $\rho_{-}(t)$. In the absence of any scheme for solving these coupled equations analytically, we turn to numerical solutions.

\section{ZENER RESONANCE WITH DISSIPATION: NUMERICAL RESULTS}

In this section we focus on the influence of dissipation on $\rho_{-}(t)$, the difference in population between the two minibands. For further simplicity the relaxation rates $\alpha_{1}$ and $\alpha_{2}$ have been set equal, $\alpha_{1}=\alpha_{2}=\alpha$, in the following.

We emphasize again that due to scattering, the time dependences of $X(k, t), Y(k, t)$ and $Z(k, t)$ as described in Eqs. (17) - (19) depend on the sum of these fyantities over all values of the wave vector, whereas in the absence of scattering, each value of $k$ is independent24,25. As we have discussed abor, we are particularly interested in the dynamics of the difference in electron population between the two minibands 29 as described by $\rho_{-}(t)$. In the perfect lattice some one-component diagonal terms $X(k, t)$ undergo Rabi oscillation 2325 — i.e., $X(k, t)$ oscillates between +1 and -1 with a period approximately determined by the value given in Ref. 23 , if we choose the electric field at a value corresponding to one of the avoided crossings of the two interpenetrating Wannier-Stark Ladders. But further analysis shows that for some other wave vectors $X(k, t)$ will not undergo complete Rabi oscillations (will not reach the full extreme values of \pm 1 ), even if we choose the electric field to be at the avoided crossings. This is depicted in the lower panels of both Figs. 1 and 2, which differ only by the choice of system parameters, listed in the figure captions. The upper panel in each corresponds to $k=0$ and the lower panel to $k=\pi$. It can be clearly seen that $X(k=\pi, t)$ falls short of the minimum value of -1 in both cases. But, for the parameters of Fig. 2, the minimum is more closely approached than it is in Fig. 1, although in Fig. 2 interband communication has been decreased. This can be understood by making the coordinate transformation $t=t^{\prime}+k / \omega_{B}$ in Eqs. (17) - (19) ( with no relaxation: $\alpha=0)$. Then all quantities depend only on the single parameter $t^{\prime}: X(k, t)=X\left(t^{\prime}\right), Y(k, t)=Y\left(t^{\prime}\right), Z(k, t)=Z\left(t^{\prime}\right)$. The initial conditions are set at a time $t$ and therefore are still $k$ dependent in terms of $t^{\prime}$. But when $E$ is very large, we have $t \approx t^{\prime}$, so that $X(k, t), Y(k, t)$, and $Z(k, t)$ will be independent of $k$. Then all $X(k, t)$ will approximately undergo full Rabi oscillations.

In general this is the dominant reason that $\rho_{-}(t)$, which represents a sum over all wave vectors, oscillates between certain negative values (but never reaches -1 ) and +1 , no matter how we choose the electric field. We demonstrate this in Fig. 3a, where we use the same parameters as those of Fig. 1. In this figure, Zener tunneling and the more rapid BO can both still be clearly identified, but some oscillations in the stable plateaus have been smeared out. In particular, when $\rho_{-}(t)$ reaches +1 , only a single sharp cusp has survived. The smearing is due to interference between the BO's for different values of $k$, which appear in slightly different positions, as can be seen by comparing the upper and lower panels of Figs. 1 and 2. This interference is also a factor (but not the main one) in suppressing the minimum of $\rho_{-}(t)$ from -1 at Zener resonances.

With the introduction of dissipation the dynamics change dramatically (even in the regime where the scattering rate is less than the Bloch frequency, $\alpha / \omega_{B}<1$, so that some BO's remain well defined; we will treat only this regime 30). We demonstrate this in Fig. 3b, where we use the same parameters as in Fig. 3a except that $\alpha / \omega_{B}=0.02$ instead of zero. In this figure, we see that $\rho_{-}(t)$ still oscillates between negative and positive values, but the oscillation amplitude decreases strikingly with increasing time. Plateaus can still be identified in several earlier peaks of the envelopes, but the BO's are increasingly suppressed in successive plateaus. If we choose the ratio $\alpha / \omega_{B}$ larger ( e.g., $\alpha / \omega_{B}=0.3$ in Fig. 3c, where we use the same other parameters as in Fig. 3a), a few BO's can be seen but $\rho_{-}(t)$ never becomes negative. This demonstrates that observation of Rabi oscillations is in general more difficult than that of BO. 
In the absence of scattering we can localize the electrons in one of the minibands simply by choosing the electric field amplitude to be well away from the avoided crossings. This can be seen through numerical calculation of $\rho_{-}(t)$. However, the introduction of dephasing destroys this picture completely. For any value of the electric field the electron density will be distributed equally between the two minibands at long times. This can be seen clearly by comparing Figs. $4 \mathrm{a}$ and $4 \mathrm{~b}$, where we use the same superlattice parameters as in Fig. 2, and the BO frequency $\omega_{B}$ has been changed from the value at an avoided crossing of $10.2 \mathrm{meV}$ to $9 \mathrm{meV}$. In Fig. 4a there is no dissipation, while in Fig. $4 \mathrm{~b}, \alpha / \omega_{B}=0.35$.

\section{PERTURBATION THEORY AND LONG TIME TRANSITIONS: EXACT RESULTS}

Under certain conditions perturbative calculations can give some useful results. Setting $A(k, t)=Y(k, t)+$ $i Z(k, t), B(k, t)=Y(k, t)-i Z(k, t), \mu \equiv 2 e E R$, and recalling $\alpha_{1}=\alpha_{2}=\alpha$, we can rewrite the Eqs. (18) and (19) as

$$
\begin{aligned}
\frac{d}{d t} A(k, t) & =-i\left[\Delta-W \cos \left(k-\omega_{B} t\right)\right] A(k, t)-\alpha A(k, t)+i \mu X(k, t), \\
\frac{d}{d t} B(k, t) & =i\left[\Delta-W \cos \left(k-\omega_{B} t\right)\right] B(k, t)-\alpha B(k, t)-i \mu X(k, t) .
\end{aligned}
$$

In order to facilitate the following perturbative manipulation, we make the transformations:

$$
A(k, t)=a(k, t) e^{-\alpha t-i \int_{0}^{t} d t^{\prime}\left[\Delta-W \cos \left(k-\omega_{B} t^{\prime}\right)\right]}
$$

and

$$
B(k, t)=b(k, t) e^{-\alpha t+i \int_{0}^{t} d t^{\prime}\left[\Delta-W \cos \left(k-\omega_{B} t^{\prime}\right)\right]}
$$

Then Eqs. (17) - (19) can be written equivalently as

$$
\begin{gathered}
\frac{d}{d t}\left[e^{\alpha t} X(k, t)\right]=-\frac{\mu}{2 i}\left[a(k, t) e^{-i \int_{0}^{t} d t^{\prime}\left[\Delta-W \cos \left(k-\omega_{B} t^{\prime}\right)\right]}-b(k, t) e^{i \int_{0}^{t} d t^{\prime}\left[\Delta-W \cos \left(k-\omega_{B} t^{\prime}\right)\right]}\right]+\alpha X_{0}(t) \\
\frac{d}{d t} a(k, t)=i \mu X(k, t) e^{\alpha t+i \int_{0}^{t} d t^{\prime}\left[\Delta-W \cos \left(k-\omega_{B} t^{\prime}\right)\right]} \\
\frac{d}{d t} b(k, t)=-i \mu X(k, t) e^{\alpha t-i \int_{0}^{t} d t^{\prime}\left[\Delta-W \cos \left(k-\omega_{B} t^{\prime}\right)\right]}
\end{gathered}
$$

As usual, we take the electrons to be initially located in band a. If the communication between the two bands is small, we can perform perturbative calculations by integrating Eqs. (25) and (26) and substituting the resultant expressions for $a(k, t)$ and $b(k, t)$ in terms of $X(k, t)$ into $(24)$, which is then integrated by iteration to the first nontrivial order. This gives, after summation over $k$, the approximate integral equation,

$$
\begin{aligned}
\rho_{-}(t)= & X_{0}(t)=e^{-\alpha t}\left\{1+\alpha \int_{0}^{t} d t^{\prime} X_{0}\left(t^{\prime}\right) e^{\alpha t^{\prime}}\right. \\
& \left.-\mu^{2} \int_{0}^{t} d t^{\prime} \int_{0}^{t^{\prime}} d t^{\prime \prime}\left[1+\alpha \int_{0}^{t^{\prime \prime}} d \tau X_{0}(\tau) e^{\alpha \tau}\right] J_{0}\left(\frac{2 W}{\omega_{B}} \sin \frac{\omega_{B}\left(t^{\prime}-t^{\prime \prime}\right)}{2}\right) \cos \Delta\left(t^{\prime}-t^{\prime \prime}\right)\right\},
\end{aligned}
$$

where $J_{0}$ is the ordinary Bessel function of order zero. Using the identity

$$
J_{0}(a \sin x)=\sum_{m} J_{m}^{2}(a / 2) e^{2 i m x},
$$

we can readily Laplace transform Eq. (27): 


$$
X_{0}(P)=\frac{1-\mu^{2} f(P+\alpha)}{P+\alpha \mu^{2} f(P+\alpha)}
$$

where $f(P+\alpha)$ is defined by

$$
f(P+\alpha)=\frac{J_{0}^{2}\left(W / \omega_{B}\right)}{(P+\alpha)^{2}+\Delta^{2}}+\sum_{m=1}^{\infty} J_{m}^{2}\left(\frac{W}{\omega_{B}}\right)\left[\frac{1}{(P+\alpha)^{2}+\left(m \omega_{B}+\Delta\right)^{2}}+\frac{1}{(P+\alpha)^{2}+\left(m \omega_{B}-\Delta\right)^{2}}\right] .
$$

Then (29) gives the long time behavior of $X_{0}(t)=\rho_{-}(t)$ as

$$
\rho_{-}(t \rightarrow \infty)=\left[1-\mu^{2} f(\alpha)\right] e^{-t / \tau},
$$

where $\tau$, the effective decay time, is defined as $\tau^{-1}=\alpha \mu^{2} f(\alpha)$. From Eqs. (30) and (31) and the definition of effective scattering time $\tau$, setting $\tau_{0}=\alpha^{-1}$, we find

$$
\frac{\tau^{-1}}{\tau_{0}^{-1}}=\left(\mu \tau_{0}\right)^{2}\left\{\frac{J_{0}^{2}\left(W / \omega_{B}\right)}{1+\left(\Delta \tau_{0}\right)^{2}}+\sum_{m=1}^{\infty} J_{m}^{2}\left(\frac{W}{\omega_{B}}\right)\left[\frac{1}{1+\left(\Delta / \omega_{B}+m\right)^{2}\left(\omega_{B} \tau_{0}\right)^{2}}+\frac{1}{1+\left(\Delta / \omega_{B}-m\right)^{2}\left(\omega_{B} \tau_{0}\right)^{2}}\right]\right\} .
$$

This relation describes the leading long time dynamics of electrons. The structure of Eq. (32) shows that when $\Delta / \omega_{B}$ is an integer, $\tau^{-1} / \tau_{0}{ }^{-1}$ will reach a local maximum; this is illustrated in Fig. 5. These resonant peaks indicate that the excited state (i.e., the rungs of the upper one of the two interpenetrating Wannier-Stark Ladders) in one well is degenerate with the ground state of a well $\mathrm{m}$ unit cells away. When the scattering rate $\alpha=\tau_{0}^{-1}$ becomes smaller, the peaks in $\tau^{-1} / \tau_{0}^{-1}$ become higher and sharper (half width in $\Delta / \omega_{B}$ of order $\left(\omega_{B} \tau_{0}\right)^{-1}=\alpha / \omega_{B}$ ), as one expects intuitively. Note that the decay time $\tau$ in the figure is always longer than the scattering time $\tau_{0}$, even at the peaks, which was tacitly assumed in setting $f(P+\alpha) \approx f(\alpha)$ at the dominant (smallest negative $P$ ) pole in (29) above.

\section{CONCLUDING REMARKS}

We have discussed the dynamics of electrons in a two-band superlattice under the influence of static electric fields, with Markoffian dephasing. We have given both analytic and numerical analyses of the effects of scattering and miniband structure on the Zener resonances and miniband localization. We found that dephasing will ultimately force the electrons initially located in one miniband to populate equally the two minibands. Under conditions of small interband coupling, and in the long time limit, we obtain the effective decay rate, whose sharp peaks are a signature of Zener resonances.

Experimentally the phenomena predicted in this paper are accessible in semiconductor superlattices with wells of dimensions on the order of 100 Angstroms and electric fields of a few kilovolts per $\mathrm{cm}$. This superlattice can be grown dimerized, with the unit cell consisting, e.g., of a pair of quantum wells, separated from the adjacent pair by a wider barrier than that which separates the two wells of the basis pair. Then the lowest level of an individual well is split within the basis pair. These two levels form a pair of minibands in the superlattice which is well separated energetically from higher levels, and all but that pair can reasonably be neglected in studying the dominant electron dynamics 2122. Then we can probe radiation induced by the oscillating dipole associated with Rabi flop in this systemes.

Work is under way to extend these results to understand the influence of scattering on the behavior of a semiconductor superlattice in the presence of strong time periodic, as well as static, electric fields. Of particular practical interest are superlattices subjected to the terahertz radiation of a free electron laser. A study of the single miniband case, including a discussion of chaotic dynamics in the presence of dissipation, has recently been published 77 by Alekseev, et al.

\section{ACKNOWLEDGMENT}

The authors thank Prof. W.-M. Zheng for useful and stimulating discussions. This work was supported in part by the National Natural Science Foundations of China and a grant of the China Academy of Engineering and Physics, and in part by the U.S. National Science Foundation Grant PHY94-07194. 
${ }^{1}$ L. Esaki and R. Tsu, IBM J. Res. Dev. 14, 61 (1970).

${ }^{2}$ F. Bloch, Z. Phys. 52, 555 (1929); A.M. Bouchard and M. Luban, Phys. Rev. B 52, 5105 (1995).

${ }^{3}$ E.E. Mendez and G. Bastard, Phys. Today 46(6), 34 (1993).

${ }^{4}$ J. Feldmann, K. Leo, J. Shah, D.A.B. Miller, J.E. Cunningham, T. Meier, G. von Plessen, A. Schulze, P. Thomas, and S. Schmitt-Rink, Phys. Rev. B 46, 7252 (1992); C. Waschke, H.G. Roskos, R. Schwedler, K. Leo, H. Kurz, and K. Köhler, Phys. Rev. Lett. 70, 3319 (1993).

${ }^{5}$ R. Tsu and G.H. Döhler, Phys. Rev. B 12, 680 (1975).

${ }^{6}$ B.J. Keay, S. Zeuner, S.J. Allen, K.D. Maranowski, A.C. Gossard, U. Bhattacharya, and M.J.W. Rodwell, Phys. Rev. Lett. 75, $4102(1995)$.

${ }^{7}$ K. Unterrainer, B.J. Keay, M.C. Wanke, S.J. Allen, D. Leonard, G. Medeiros-Ribeiro, U. Bhattacharya, and M.J.W. Rodwell, Phys. Rev. Lett. 76, 2973 (1996).

${ }^{8}$ D.H. Dunlap and V.M. Kenkre, Phys. Rev. B 34, 3625 (1986).

${ }^{9}$ D.H. Dunlap and V.M. Kenkre, Phys. Lett. A 127, 438 (1988).

10 N.H. Shon and H.N. Nazareno, J. Phys: Condens. Matter 4, L611 (1992).

${ }^{11}$ X.-G. Zhao, Phys. Lett. A 155, 299 (1991); 167, 291 (1992).

12 T. Meier, G. von Plessen, P. Thomas, and S.W. Koch, Phys. Rev. Lett. 73, 902 (1994); Phys. Rev. B 51, 14490 (1995).

${ }^{13}$ M. Holthaus, G.H. Ristow, and D.W. Hone, Europhys. Lett. 32, 241(1995).

${ }^{14}$ M. Holthaus, Phys. Rev. Lett. 69, 351 (1992).

15 J. Zak, Phys. Rev. Lett. 71, 2623 (1993).

${ }^{16}$ D.W. Hone and M. Holthaus, Phys. Rev. B 48, 15123 (1993).

${ }^{17}$ X.-G. Zhao, J. Phys: Condens. Matter 6, 2751 (1994).

18 X.-G. Zhao, R. Jahnke, and Q. Niu, Phys. Lett. A 202, 297 (1995).

19 B.S. Monozon, J.L. Dunn and C.A. Bates, Phys. Rev. B 50, 17097 (1994); B.S. Monozon, J.L. Dunn, and C.A. Bates, J. Phys.:Condens. Matter 8, 877 (1996); B.S. Monozon, J.L. Dunn, and C.A. Bates, J. Phys.: Condens Matter, 6, 4009 (1994).

${ }^{20}$ H. Fukuyama, R.A. Bari, and H.C. Fogedby, Phys. Rev. B 8, 5579 (1973).

${ }^{21}$ M. Holthaus and D.W. Hone, Phys. Rev. B 49, 16605 (1994).

${ }^{22}$ D.W. Hone and X.-G. Zhao, Phys. Rev. B 53, 4834 (1996).

${ }^{23}$ X.-G. Zhao, G.A. Georgakis, and Q. Niu, Phys. Rev. B 54, R5235 (1996).

${ }^{24}$ J. Rotvig, A.-P. Jauho, and H. Smith, Phys. Rev. Lett. 74, 1831 (1995).

25 J. Rotvig, A.-P. Jauho, and H. Smith, Phys. Rev. B 54 , 17691 (1996).

${ }^{26}$ For the single band system this approach was analyzed by D.H. Dunlap and V.M. Kenkre, Phys. Rev. B 34, 6622 (1988).

${ }^{27}$ K.N. Alekseev, G.P. Berman, D.K. Campbell, E.H. Cannon, and M.C. Cargo, Phys. Rev. B 54, 10625 (1996).

${ }^{28}$ J.B. Krieger and G.J. Iafrate, Phys. Rev. B 33, 5494 (1986).

${ }^{29}$ S.T. Cundiff, A. Knorr, J. Feldmann, S.W. Koch-E.O. Göbel, and H. Nickel, Phys. Rev. Lett. 73, 1178 (1994).

${ }^{30}$ The very observation of $\mathrm{BO}$, and measurements 31 of cyclotron resonance linewidths suggest as reasonable values for $\alpha$ in practical semiconductor superlattices something on of the order of a terahertz. Then for a typical lattice constant $d$ of 100 Angstroms the Bloch frequency $\omega_{B}=e E d$ becomes equal to $\alpha$ for electric fields $E$ of order $1 \mathrm{kV} / \mathrm{cm}$.

${ }^{31}$ T. Duffield, et al., Sol. State Commun. 65, 1483 (1988). 
FIG. 1. Dependence on wave vector $k$ of both oscillation amplitudes and the position of BO of a single component $X(k, t)$, plotted as functions of dimensionless time, $\omega_{B} t$. In the upper panel (with $k=0$ ), full Rabi oscillations can be clearly seen, while in the lower panel (with $k=\pi$ ), they are incomplete. In both figures $W=\left(W_{a}+W_{b}\right) / 2=18 \mathrm{meV}, \Delta=\Delta_{a}-\Delta_{b}=20$ $\mathrm{meV}, e E R=2.09 \mathrm{meV}$, and $\omega_{B}=2.32 \mathrm{meV}$ is at an avoided crossing of the interpenetrating WSL from the two bands.

FIG. 2. The same as in Fig. 1, with $W=8.6 \mathrm{meV}, \Delta=20 \mathrm{meV}, e E R=1.84 \mathrm{meV}$, and $\omega_{B}$ has been increased to $10.2 \mathrm{meV}$ (still at an avoided crossing).

FIG. 3. The density matrix $\rho_{-}(t)$, with and without dissipation. We use the same superlattice parameters as those of Fig. 1. (a) no scattering, $\alpha / \omega_{B}=0$; (b) small scattering rate, $\alpha / \omega_{B}=0.02$; (c) large scattering rate, $\alpha / \omega_{B}=0.3$.

FIG. 4. The effect of scattering on miniband localization. (a) no scattering; (b) the complete destruction of localization by dephasing (with $\alpha / \omega_{B}=0.35$ ). Here $W=\left(W_{a}+W_{b}\right) / 2=8.6 \mathrm{meV}, \Delta=\Delta_{a}-\Delta_{b}=20 \mathrm{meV}$, eER=1.84 meV, and $\omega_{B}=9$ meV.

FIG. 5. Long time decay rate $\tau^{-1} / \tau_{0}^{-1}$ of $\rho_{-}(t)$ (see Eqs. (31) and (32)). The local maxima appear when $\Delta / \omega_{B}$ is an integer. Here $\mu / \omega_{B}=0.18, \alpha / \omega_{B}=0.2$, and $W / \omega_{B}=1.3$. 

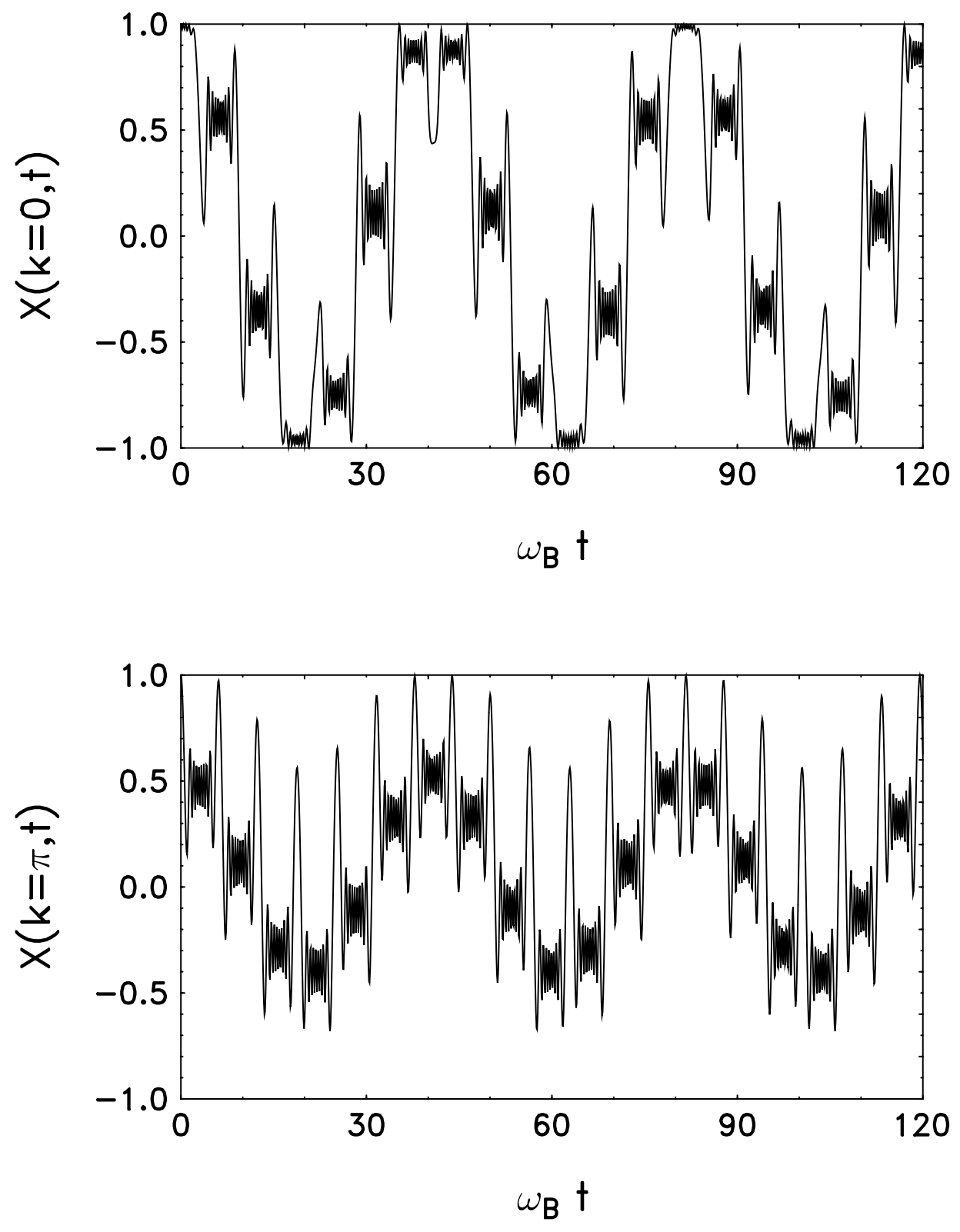

Fig. 1 

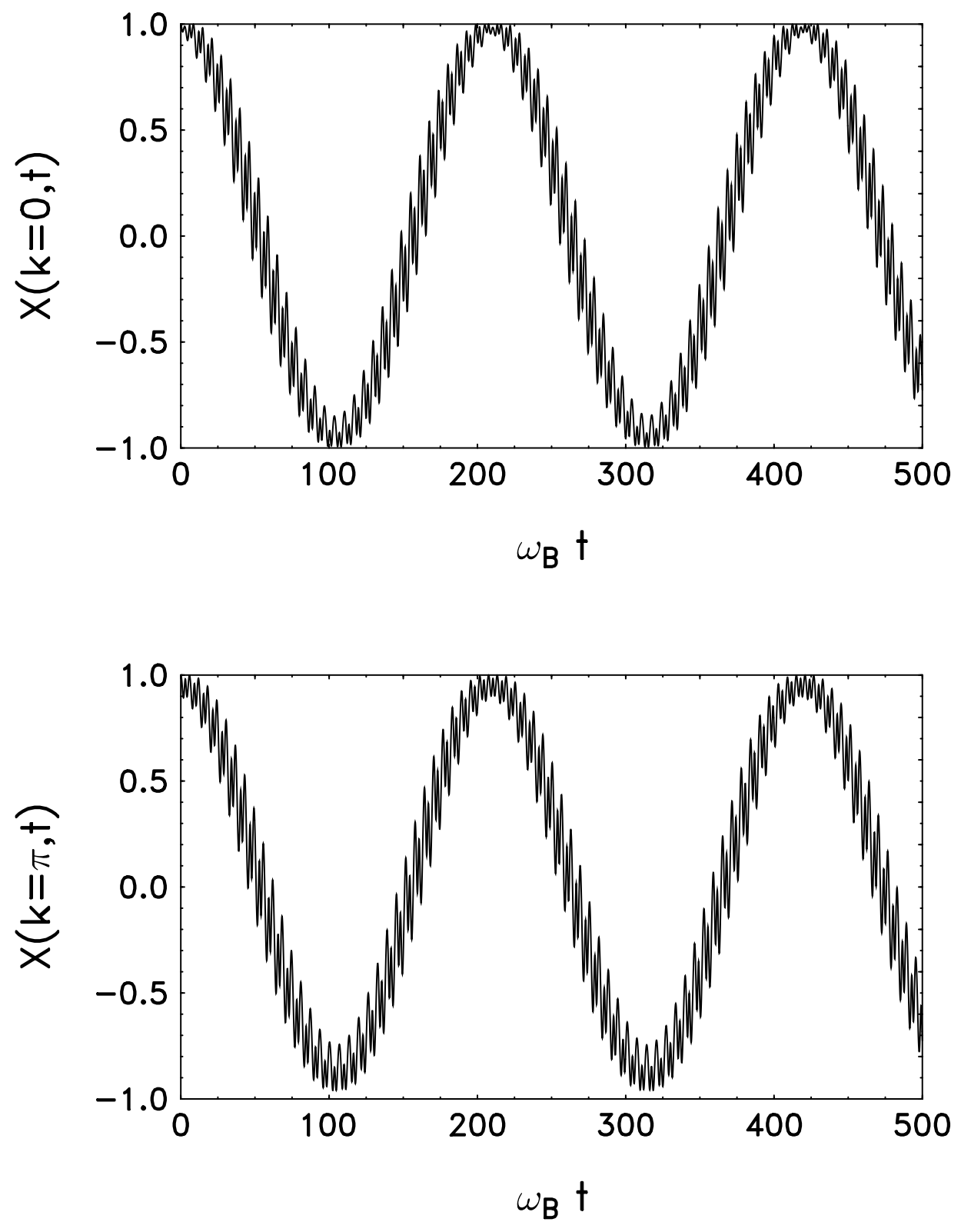

Fig. 2 

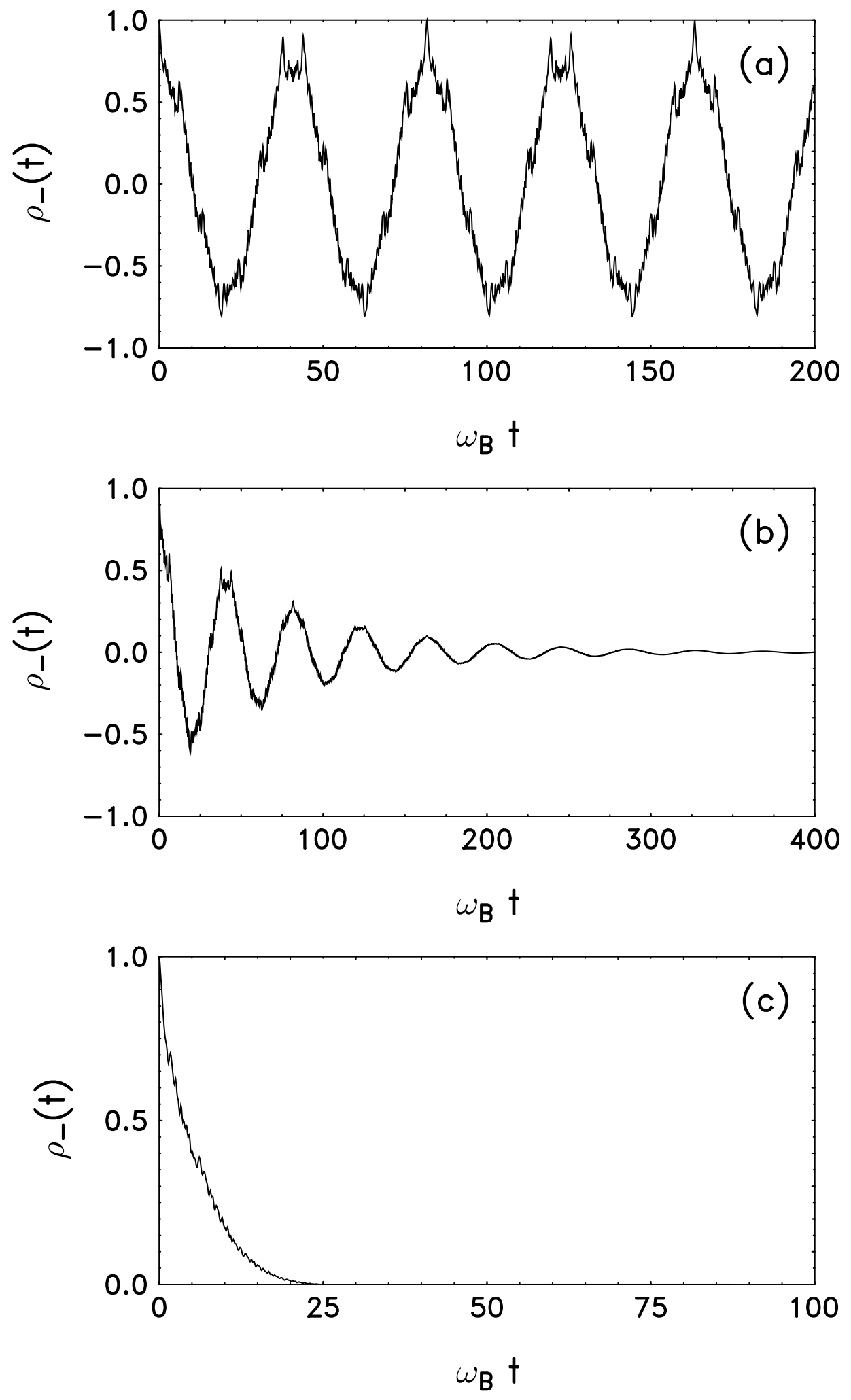

Fig. 3 

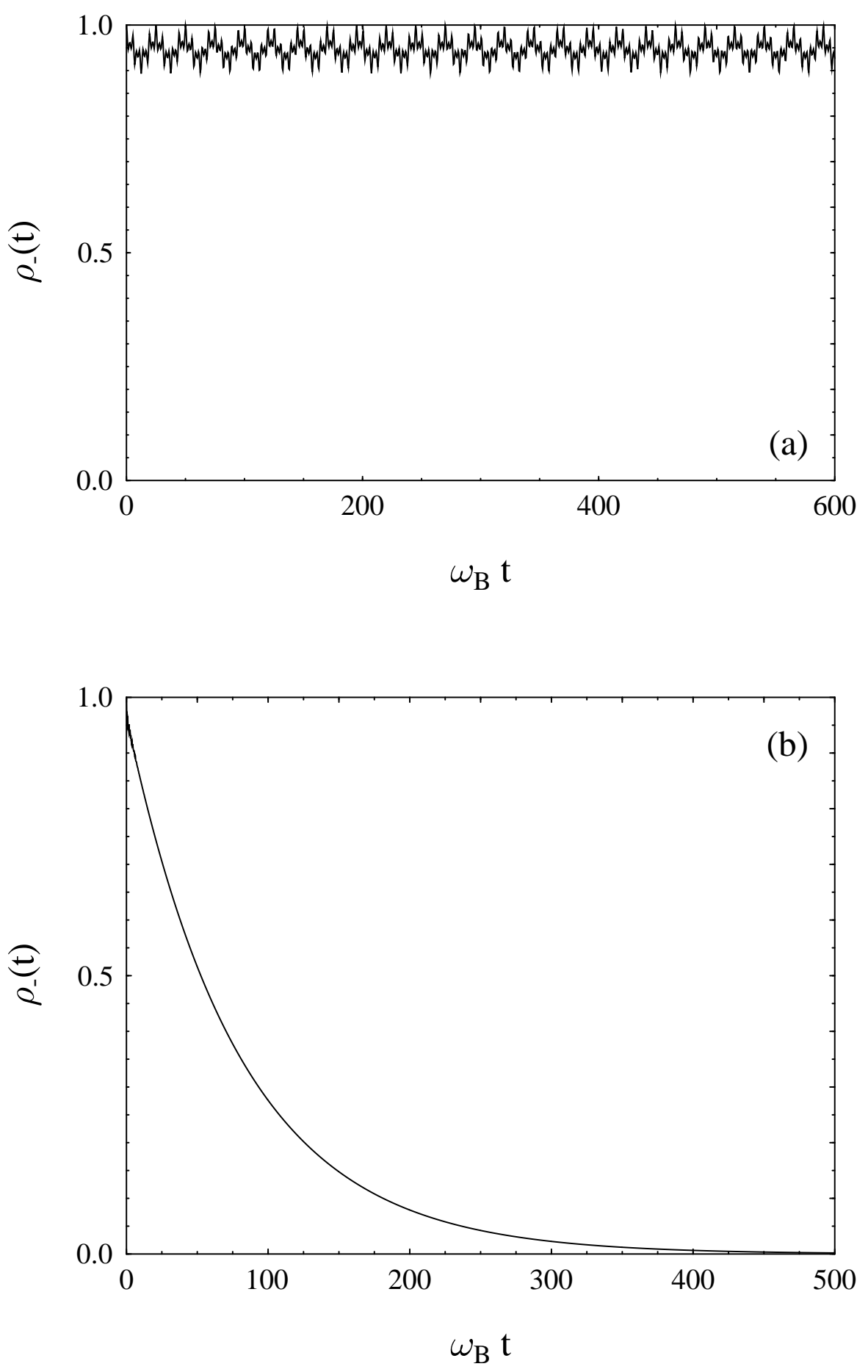

Fig. 4 


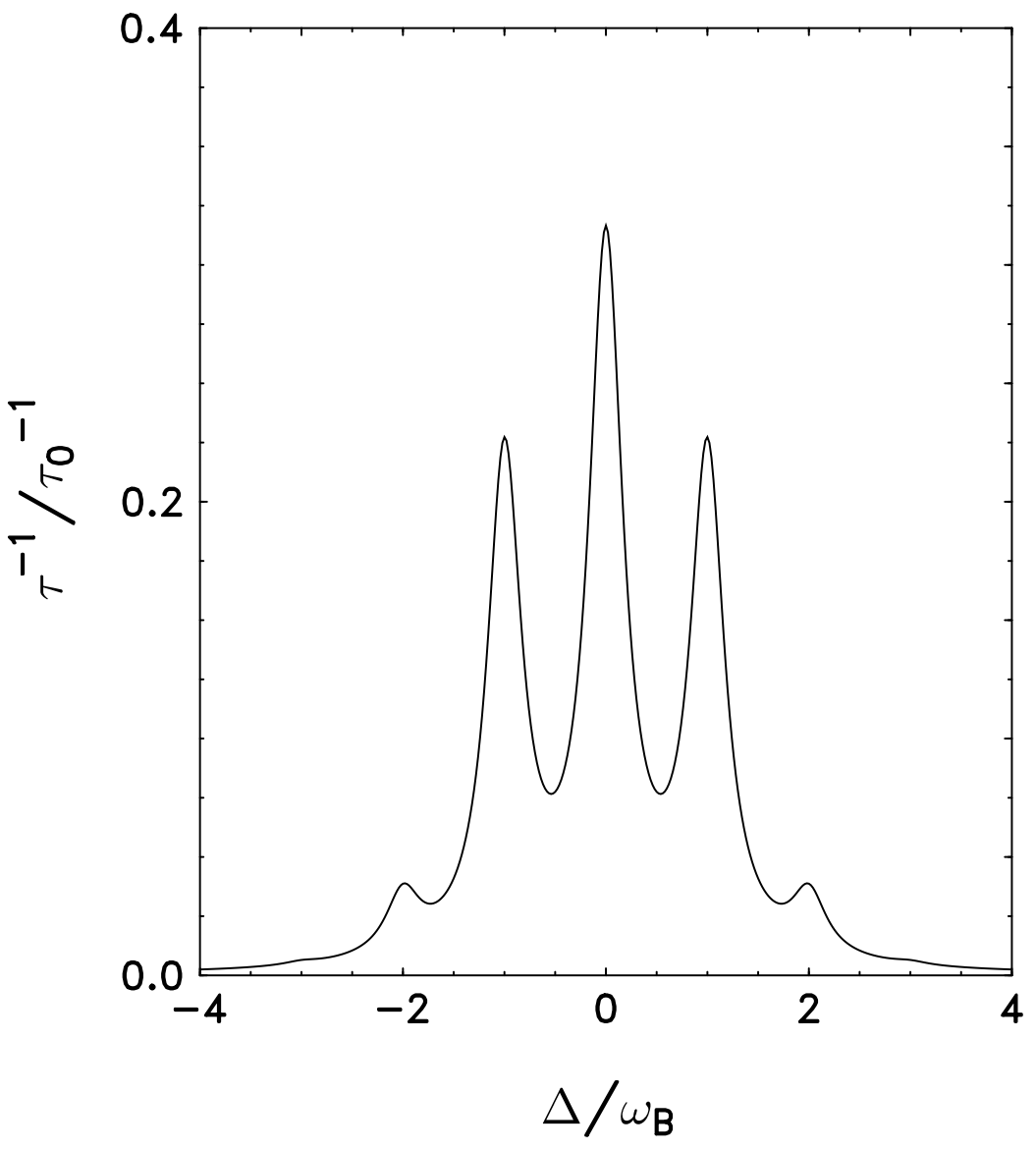

Fig. 5 